\title{
Echocardiographic Parameters Predict Short- and Long-Term Adverse Cardiovascular Events in Patients with Acute Myocardial Infarction
}

\author{
Meng-Meng $\operatorname{Han}^{1} * *$ \\ Wen-Shu Zhao ${ }^{2} *$ \\ Xin Wang ${ }^{2, *}$ \\ Shan $\mathrm{He}^{3}$ \\ Xiao-Rong $\mathrm{Xu}^{2}$ \\ Cai-Jing Dang ${ }^{2}$ \\ Juan Zhang ${ }^{2}$ \\ Jia-Mei Liu ${ }^{2}$ \\ Mu-Lei Chen ${ }^{2}$ \\ Lin $X u^{2}$ \\ Hua Wang ${ }^{2}$
}

'Intensive Care Units, Beijing Longfu Hospital, Beijing, 100010, People's

Republic of China; ${ }^{2}$ Heart Center and Beijing Key Laboratory of Hypertension Research, Beijing Chaoyang Hospital, Capital Medical University, Beijing, 100020, People's Republic of China; ${ }^{3}$ Heart Center, Beijing Chaoyang Hospital Jingxi Branch, Capital Medical University, Beijing, 100020, People's Republic of China

*These authors contributed equally to this work

Correspondence: Lin Xu; Hua Wang Heart Center and Beijing Key Laboratory of Hypertension Research, Beijing Chaoyang Hospital, Capital Medical University, Beijing, 100020, People's Republic of China

Tel +86-I0-8523 I445; +86-I0-8523I556

Email Ixdrqw09_dr@।63.com;

huwsmndr66@yandex.com
Objective: This study aimed to find echocardiographic parameters that can predict shortand long-term adverse cardiovascular events in patients with AMI.

Methods: A total of 126 patients with AMI admitted to our hospital from July to December 2012 were enrolled in this study. All patients underwent echocardiographic examination within 12 hours after admission and received regular follow-ups until December 2018. The primary endpoint was a composite of the major adverse cardiovascular events (MACEs).

Results: In the first year of this study, a primary endpoint occurred in 35 patients and the predictor derived from the echocardiography of 1-year primary endpoint was LVEF $<40 \%$ (OR: 9.000, 95\% CI 3.242-24.987, $<<0.0001$ ) and the area under the curve (AUC) for the predictor was $0.676(95 \%$ CI $0.561-0.790, \mathrm{p}=0.002)$. For the total 5 years, 57 patients underwent primary endpoint. The results of the 5-year primary endpoint were: E/E'>15 (OR: 4.094, 95\% CI 1.726-9.710, $\mathrm{P}=0.001$ ), the wall motion score index was (WMSI) $>1.5$ (OR: $12.791,95 \%$ CI 1.511-108.312, $\mathrm{P}=0.019)$, and the AUC was 0.691 (95\% CI 0.595 $0.787 \mathrm{P}<0.0001)$.

Conclusion: LVEF is correlated with a short-term outcome (1-year), and WMSI and E/E' can predict a long-term outcome (5-year) in patients with acute myocardial infarction.

Keywords: echocardiography, cardiovascular events, predictors, acute myocardial infarction, wall motion score index

\section{Introduction}

Acute myocardial infarction (AMI) is one of the most serious life-threatening conditions. The most important parameter to determine the outcome of AMI is the occurrence of a major adverse cardiovascular event (MACE). MACE includes various cardiovascular events, such as myocardial infarction, unstable angina, cardiovascular death, revascularization, fatal/nonfatal cerebrovascular accident, peripheral arteriopathy, aortic event, etc. ${ }^{1}$ Various prognostic tools are developed and implemented in clinics to predict the occurrence of MACE. For example, biomarkers are one of the most commonly used methods. It includes troponin, which is very sensitive and specific for AMI and can independently predict adverse cardiovascular events. ${ }^{2}$ Other biomarkers such as N-terminal B-type natriuretic peptide and $\mathrm{C}$ reactive protein are also valuable in the prognosis of AMI. ${ }^{2}$ However, biomarkers often vary among individuals and so it is not always the best practice to use biomarkers to predict MACE. 
Echocardiography plays a fundamental role in risk stratification and predicting the outcome in patients with acute myocardial infarction (AMI). ${ }^{3}$ Both American and European guidelines recommended echocardiography as the elective cardiac imaging modality for identifying patients with AMI at high risk for adverse short-term and long-term cardiovascular events, in particular, reinfarction and death. ${ }^{4}$ Although echocardiography (the most frequently used cardiovascular diagnostic method besides electrocardiography in AMI) is used to detect myocardial walls involved in the ischemic process, damage extent, functional consequences, and mechanical complications in patients after AMI and can provide prognostic information on short- and long-term outcomes, ${ }^{5,6}$ previous studies have shown inconsistent predictors of echocardiography and there were no studies focusing on the echocardiographic parameters for predicting the short- and longterm prognosis of patients with AMI. ${ }^{7}$

Although a variety of individual echocardiographic parameters were known to be associated with short-term outcomes, multiple factors must be taken into account simultaneously in a multivariable model to quantify their predictive ability. The purpose of this study is to investigate the predictive value of multiple echocardiographic parameters for early identifying patients at high risk for developing short- and long-term adverse events after AMI.

\section{Methods}

\section{Study Population}

One hundred and twenty-six AMI patients of Beijing Chao-Yang Hospital were enrolled from July to December 2012 in this study. All patients underwent emergency coronary angiography within 12 hours after the onset of their symptoms. Of them 81 patients were treated with primary PCI. All patients received standard pharmacological therapy (Aspirin, Clopidogrel, Angiotensinconverting enzyme inhibitors (ACEI)/Angiotensin receptor blockers (ARB), $\beta$-blockers and statin, except when these agents were contraindicated).

The inclusion criteria were as follows: 1.The typical chest pain continued for over 30 minutes; 2. Hospital admission was within 12 hours of the onset of symptoms; 3.The electrocardiogram (ECG) were collected; 4 . The patients received elevated serum troponin (cTnI) and creatine phosphokinase myocardial band (CKMB) more than twice for the upper normal limit; and 5. The patients were able to complete the study and follow-ups.
The exclusion criteria included the presence of any one of the following conditions: 1 . Mechanical difficulties after AMI including a free wall rupture, ventricular septal perforation, and papillary muscle break; 2. Cardiogenic shock; 3. Cardiomyopathy and valvular heart disease; 4 . Severe hepatic and renal dysfunction; and 5. Terminal disease with a predicted survival time of $<1$ year (eg terminal cancer).

The study adhered to the Declaration of Helsinki and was approved by the Ethics Committee and the Prescription and Therapeutic Committee of Beijing ChaoYang Hospital, Capital Medical University. All the patients gave informed consent before the study started.

\section{Clinical Data}

The patients' clinical data, including age, gender, height, weight, body mass index (BMI), body surface area (BSA), systolic blood pressure (SBP), diastolic blood pressure (DBP), heart rate (HR), type of AMI, cardiac function after AMI, the results of invasive coronary angiography, and treatment were recorded upon the enrollment in this study.

\section{Echocardiographic Data}

All patients underwent a routine echocardiographic examination within 12 hours after admission in our hospital. Standard transthoracic echocardiography was performed according to the guidelines of the American Society of Echocardiography recommendations. ${ }^{7}$ All analyses were performed by experienced sonographers and interpreted by a single experienced physician.

\section{The Follow-Up and Endpoint Events}

We followed-up on patients using outpatient visits or telephone contact about heart failure symptoms, rehospitalization, lab examination and echocardiography. Each patient was assigned to one designated study investigator from whom the patient received the follow-up examination at least once per month for 12 months after the start of our study, and then every 3-6 months until the primary endpoint. We followed-up on patients using outpatient visits or telephone contact from January 2013 to December 2018. The primary endpoint events were a composite of the major adverse cardiovascular events (MACEs) including re-hospitalization for unstable angina, reinfarction, heart failure, and all-cause mortality. Heart failure was diagnosed based on symptoms (eg, dyspnea, fatigue, or decreased exercise capacity), signs (eg, edema or rales) and B-type natriuretic peptide (BNP) $>35 \mathrm{pg} / \mathrm{mL}$ 
or N-terminal pro-B-type natriuretic peptide (NT-proBNP) $>125 \mathrm{pg} / \mathrm{mL}$. Heart failure with reduced ejection fraction (HFrEF) and preserved ejection fraction (HFpEF) were predefined as left ventricular ejection fraction (LVEF) $<40 \%$ and $\geq 40 \%$. The members of an independent endpoint committee that were blind to the study group assignments and used pre-specified criteria adjudicated all the endpoint events.

\section{Statistical Analysis}

Continuous variables were presented as mean \pm standard deviation (SD). Categorical variables were displayed as frequency and percentages. The comparisons between the groups were completed by a $t$-test for continuous variables and a chi-square test for categorical variables. All-cause mortality was evaluated using the Cox proportional hazards model. Logistic regression analysis was used to assess predictors of endpoint events. Receiver-operator curves (ROC) for predictors were generated from Logistic regression. The covariates included LVEF, LVESD, LVESV, LVESVI, E', E/E', E/A, and WMSI. $\mathrm{P}<0.05$ was considered statistically significant. SPSS 22.0 software was used for the statistical analyses.

\section{Results}

\section{The Baseline Characteristics and Adverse Events}

The characteristics and echocardiographic data of the study population are summarized in Table 1. The mean LEVF was $53 \pm 14 \%$ and the means LVESD, LVESV, LVESVI, E', E/E', and WMSI were $32 \pm 7 \mathrm{~mm}, 38 \pm 20 \mathrm{~mL}, 21 \pm 11 \mathrm{~mL} / \mathrm{m}^{2}, 6.3$ $\pm 1.8 \mathrm{~cm} / \mathrm{s}, 13 \pm 5$, and $1.2 \pm 0.2$, respectively.

During a mean follow-up of $51 \pm 15.4$ months, all patients were contacted in the first year and $5(3.9 \%)$ of the patients were absent in the fifth year of the study.

In the first year, the primary endpoint occurred in 35 patients, including 6 deaths, 8 cases of reinfarction, 14 cases of heart failure, and 7 cases of re-hospitalization for additional reasons. However, in the fifth year, 57 patients underwent primary endpoint events, including 23 deaths, 12 cases of reinfarction, 11 cases of heart failure, and 11 cases of re-hospitalization for additional reasons.

\section{The Univariate and Multivariate Predictors of I-Year MACEs}

In the first year, univariate variables by Logistic regression were significantly correlated with primary endpoint events including LVEF, LVESD, LVESV, LVESVI, E', and WMSI. All significant univariate variables were recorded in the multivariable Logistic regression model and only LVEF was identified as an independent predictor of 1-year primary endpoint events, as shown in Table 2.

The area under the curve (AUC) of the ROC curve for LVEF $<40 \%$ was 0.676 (95\% CI 0.561-0.790, $\mathrm{P}=0.002$ ), which meant that $\mathrm{LVEF}<40 \%$ had a good predictive value for 1-year primary endpoint events, as shown in Figure 1A.

\section{The Univariate and Multivariate Predictors of 5-Year MACEs}

For the fifth year, 5 patients did not continue the followups and 121 patients were systematically examined. Using the univariate Logistic regression analyses, the following variables were potential contributors: $\operatorname{LVEF}<40 \%$, LVESD, LVESV, LVESVI, E', E/E'>15, E/A>2, and WMSI $>1.5$. After adjusting for the covariates, E/E'>15 and WMSI $>1.5$ were recognized as predictors of 5-year primary endpoint events in AMI, as shown in Table 2.

The AUC of ROC for E/E'>15 was $0.642(95 \%$ CI $0.542-0.742, \mathrm{P}=0.007)$ and $\mathrm{WMSI}>1.5$ was $0.580(95 \% \mathrm{CI}$ $0.477-0.683, \mathrm{P}<0.0001)$. The AUC of ROC for these two predictors was 0.691 (95\% CI $0.595-0.787 \mathrm{P}<0.0001)$, showing a good predicting capacity of 5 -year primary endpoint events in AMI, as shown in Figure 1B-D.

\section{Discussion}

To the best of our knowledge, this is the first study to report the multiple echocardiographic parameters predicting shortand long-term outcomes in the same population. The significant findings of our study revealed that $\mathrm{LVEF}<40 \%$ was related to 1-year primary endpoint events, however, when it came to the 5-year primary endpoint events, the independent risk predictors were $\mathrm{E} / \mathrm{E}^{\prime}>15$ and WMSI $>1.5$.

The left ventricular ejection fraction (LVEF) was the most commonly used parameter to assess the left ventricular (LV) function and provides important prognostic information after the onset of AMI. ${ }^{8}$ Reduced LVEF was also a predictor of sudden cardiac death (SCD) in myocardial infarction. Lower LVEF might be the result of reduced contractile function due to extensive myocardial damage or continuing ischemia. Moller et $\mathrm{al}^{9}$ demonstrated that echocardiographically determined LVEF at 1 day after admission for AMI was a powerful predictor of all-cause mortality during a median follow-up period of 19 months. The mortality curve after the onset of AMI 
Table I Baseline Demographic and Echocardiographic Data of the Study Population

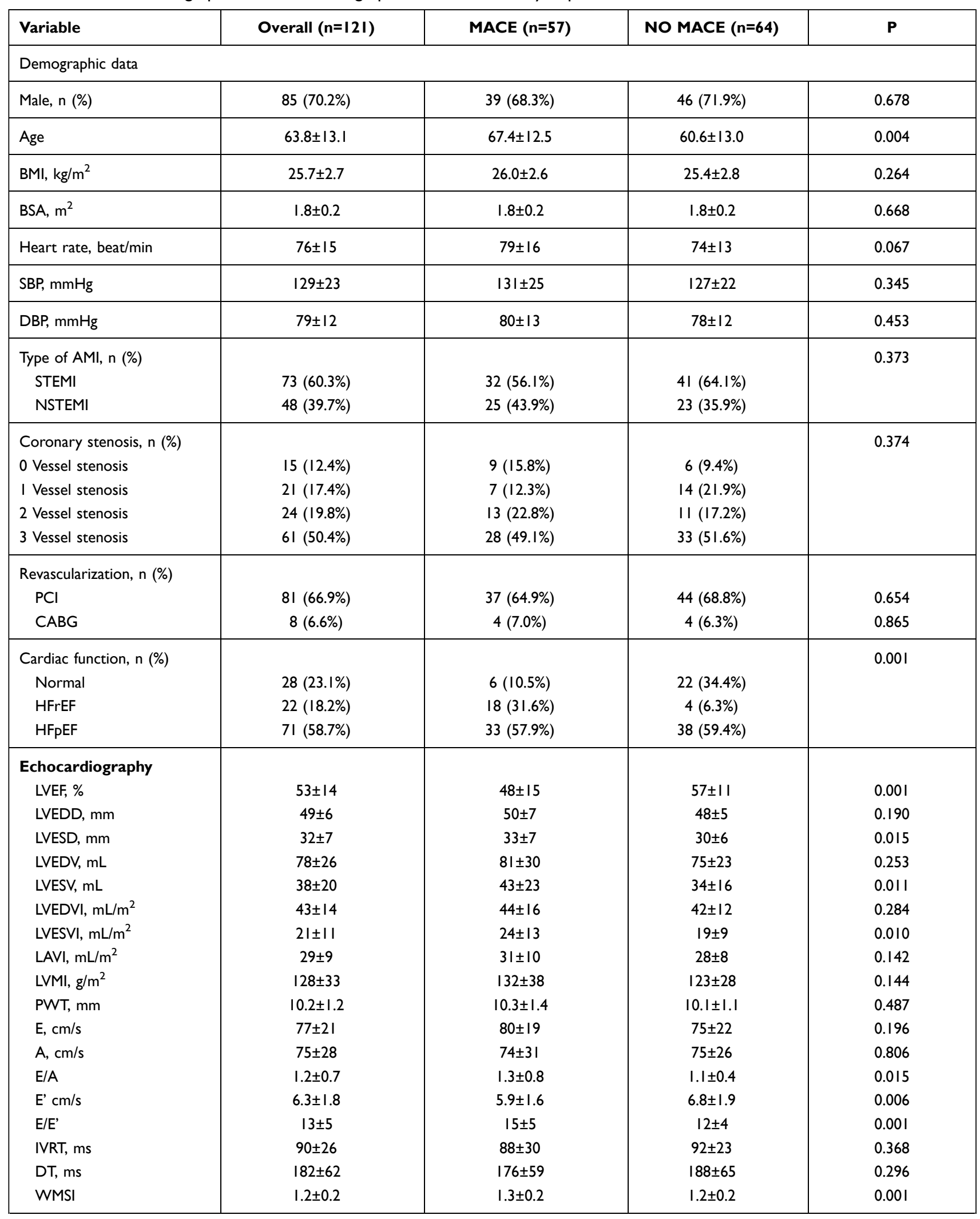


Table I (Continued).

\begin{tabular}{|c|c|c|c|c|}
\hline Variable & Overall $(n=|2|)$ & MACE $(n=57)$ & NO MACE $(n=64)$ & $\mathbf{P}$ \\
\hline \multicolumn{5}{|c|}{ Medicines in hospital, n (\%) } \\
\hline Aspirin & $116(95.9 \%)$ & 52 (91.2\%) & $64(100 \%)$ & 0.016 \\
\hline Clopidogrel & 109 (90.1\%) & $53(93.0 \%)$ & $56(87.5 \%)$ & 0.314 \\
\hline ACEI/ARB & 65 (53.7\%) & 29 (50.9\%) & $36(56.2 \%)$ & 0.554 \\
\hline Metoprolol & $83(68.6 \%)$ & 35 (61.4\%) & $48(76.2 \%)$ & 0.108 \\
\hline Statin & $104(86.0 \%)$ & 48 (84.2\%) & 57 (88.1\%) & 0.603 \\
\hline Nitrate & 78 (64.5\%) & 34 (59.6\%) & 44 (68.3\%) & 0.296 \\
\hline $\mathrm{CCB}$ & $20(16.5 \%)$ & II (19.3\%) & $9(14.3 \%)$ & 0.439 \\
\hline Diuretic & $19(15.7 \%)$ & $15(26.3 \%)$ & $4(6.3 \%)$ & 0.002 \\
\hline MRA & 15 (12.4\%) & $12(21.1 \%)$ & $3(4.7 \%)$ & 0.006 \\
\hline Digoxin & $4(3.3 \%)$ & $3(5.3 \%)$ & I (I.6\%) & 0.256 \\
\hline
\end{tabular}

Abbreviations: BMI, body mass index; BSA, body surface area; SBP, systolic blood pressure; DBP, diastolic blood pressure; STEMI, ST-segment elevation infarction; NSTEMI, non-ST-segment elevation infarction; $\mathrm{PCl}$, percutaneous coronary intervention; $\mathrm{CABG}$, coronary artery bypass grafting; HFrEF,heart failure with reduced ejection fraction; HFpEF, heart failure with preserved ejection fraction; LVEF, left ventricular ejection fraction; LVEDD, left ventricular end diastolic diameter; LVESD, left ventricular end systolic diameter; LVEDV, left ventricular end diastolic volume; LVESV, left ventricular end systolic volume; LVEDVI, left ventricular end diastolic volume index; LVESVI, left ventricular end systolic volume index; LVMI, left ventricular mass index; PWT, posterior wall thickness; E, early diastolic transmitral inflow velocity; A, late diastolic transmitral inflow velocity; E', early diastolic transmitral annulus velocity; LAVI, left atrial volume index; IVRT, Isovolumic relaxation time; WMSI, wall motion score index.

Table 2 Univariate and Multivariate Predictors of I-Year and 5-Year MACE Events

\begin{tabular}{|c|c|c|c|c|c|c|}
\hline & \multicolumn{2}{|c|}{ Univariate } & \multirow[b]{2}{*}{$\mathbf{P}$} & \multicolumn{2}{|c|}{ Multivariate } & \multirow[b]{2}{*}{$\mathbf{P}$} \\
\hline & OR & $95 \% \mathrm{Cl}$ & & OR & $95 \% \mathrm{Cl}$ & \\
\hline \multicolumn{7}{|c|}{ I-year MACE } \\
\hline LVEF $<40 \%$ & 9.000 & $3.242-24.987$ & $<0.0001$ & 9.000 & $3.242-24.987$ & $<0.0001$ \\
\hline LVESD & I.09| & $1.025-1.163$ & 0.007 & & & \\
\hline LVESV & 1.032 & $1.011-1.054$ & 0.003 & & & \\
\hline LVESVI & 1.060 & $1.020-1.100$ & 0.003 & & & \\
\hline $\mathrm{E}^{\prime}$ & 0.718 & $0.553-0.932$ & 0.013 & & & \\
\hline WMSI>I.5 & 15.404 & $3.13 \mid-75.783$ & 0.001 & & & \\
\hline \multicolumn{7}{|c|}{ 5-year MACE } \\
\hline LVEF $<40 \%$ & 6.923 & $2.179-21.996$ & 0.001 & & & \\
\hline LVESD & 1.074 & $1.012-1.139$ & 0.018 & & & \\
\hline LVESV & 1.026 & $1.005-1.047$ & 0.016 & & & \\
\hline LVESVI & 1.048 & $1.009-1.088$ & 0.014 & & & \\
\hline $\mathrm{E}^{\prime}$ & $0.74 I$ & $0.592-0.926$ & 0.008 & & & \\
\hline$E / E^{\prime}>15$ & 4.041 & $1.757-9.292$ & 0.001 & 4.094 & $1.726-9.710$ & 0.001 \\
\hline$E / A>2$ & 9.187 & I.093-77.209 & 0.041 & & & \\
\hline WMSI>I.5 & 13.404 & $1.658-108.378$ & 0.015 & $|2.79|$ & $1.511-108.312$ & 0.019 \\
\hline
\end{tabular}

exhibited a typical increase with an upturn in mortality occurring at LVEF $<40 \%{ }^{9,10}$ Schwammenthal et al ${ }^{11}$ showed that, in patients with AMI, LVEF $<40 \%$ was an independent predictor of the combined endpoint of death, congestive heart failure, and recurrent AMI at 30 days after the treatment of AMI. Similar to previous studies, our results also indicated that $\mathrm{LVEF}<40 \%$ was a powerful determination of 1-year primary endpoint in patients with AMI. The reason for these similar results of LVEF may be that patients with $\mathrm{LVEF}<40 \%$ may have more serious myocardial damage or may experience continuing ischemia; thus, they have a higher risk of sudden cardiac death. Consequently, $\mathrm{LVEF}<40 \%$ can predict short-term outcomes, specifically within 1 year and patients with a result lower than $\mathrm{LVEF}<40 \%$ should be followed up closely for 1-year MACE. The intensive medical management of patients with reduced LVEF should also be completed to prevent the development of MACEs. 

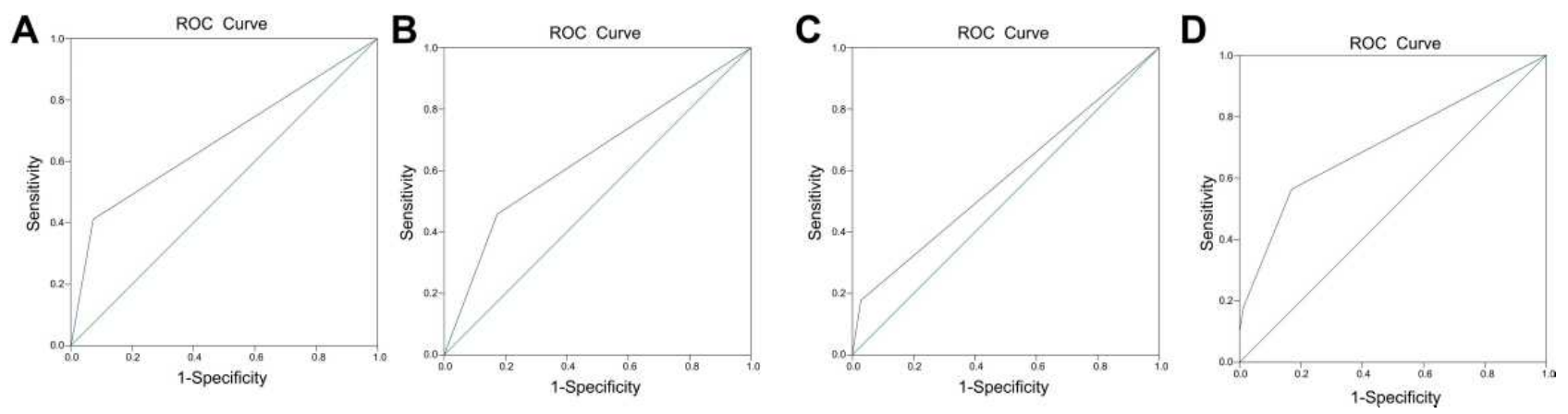

Figure I (A) The ROC curve for the predictor of I-year MACEs. (B-D) The ROC of predictors in the 5-year follow-up. (B) The ROC curve for predictors of E/E>I.5. (C) The ROC curve for the predictor of WMSI>I.5. (D) The ROC curve for predictors of the 5-year MACEs.

The wall motion score index (WMSI), a score index that evaluates the segmental function of the left ventricular, was supposed to be superior to LVEF in identifying patients who had a mild left ventricular dysfunction. ${ }^{12}$ After the onset of AMI, extensive regional wall motion abnormalities may be present but may be compensated by the regional hyperkinesis of normal segments, so LVEF could be normal. Baron et $\mathrm{al}^{12}$ showed that both LVEF and WMSI were powerful predictors of adverse cardiovascular events, however, in the multivariate model, WMSI $>1.8$ was proved to be the most powerful predictor. There is a good correlation between the wall motion score index and contractile functional impairment: a wall motion score index of 1.1-1.9 can predict small infarct size and an index equal to or greater than 2.0 can predict the occurrence of complications. Carluccio et $\mathrm{al}^{13}$ also showed that WMSI $>1.5$ was the most powerful predictors that included LVEF and LVESV for adverse cardiac events. Our data also supported the results that WMSI $>1.5$ was associated with the 5-year primary endpoint events. In patients with AMI, WMSI could reflect the magnitude of myocardial damage more correctly. ${ }^{14}$

The ratio of early transmitral flow velocity to spectral pulse wave Tissue Doppler imaging (TDI) showed that early diastolic velocity of mitral annulus (E/E') was correlated accurately with the LV filling pressures degree and is a good predictor of the prognosis after the onset of AMI. ${ }^{15}$ Our results indicated that E/E' $>15$ was related to 5-year MACEs. The E/E' ratio should be used to estimate LV filling pressure in AMI patients with impaired or normal LVEF when the transmitral pattern was nonrestrictive and increased LV filling pressure cannot be diagnosed by the simple transmitral flow assessment. It was also strongly promoted by the American Society of Echocardiography and the European Association of
Cardiovascular Imaging recommendations on the diastolic function in relation to its high feasibility. ${ }^{16,17}$

Based on our results and previous studies, the reason why WMSI and E/E' are superior to LEVF for the long-term prognosis of AMI may be explained as the following: After the onset of AMI, extensive regional wall motion abnormalities may be present but may be compensated by the regional hyperkinesis of normal segments, so LVEF could be normal. WMSI was supposed to be superior to LVEF in identifying patients who had mild left ventricular dysfunction. WMSI was associated with mild systolic function and $\mathrm{E} / \mathrm{E}$ ' assessed diastolic function. Patients with these two abnormal parameters might have a relatively lower risk for adverse cardiovascular events in the short-term but were negatively related to longterm outcomes, which could be used to predict long-term outcomes.

The limitations of this study are as follows: Firstly, our study only enrolled 126 patients with AMI, so the number of events may be relatively small in excluding the moderate associations of some variables that were tested to assess the outcomes. Secondly, LVEF, E/E', and WMSI could significantly change after complete revascularization and would be less predictive when they are measured before complete revascularization. Thirdly, we could not collect complete medical information during the follow-ups since many patients could not provide their accurate medicine use.

\section{Conclusion}

LVEF $<40 \%$ was associated with short-term outcomes and WMSI $>1.5$ and $E / E^{\prime}>1.5$ were associated with long-term prognoses. These indicators may be potential predictors for estimating the prognosis of patients with AMI. The practical advantage of these findings is that these simple echocardiographic parameters can be easily obtained without the use of complex analysis methods. 


\section{Funding}

This project was supported by the Atherosclerosis Special Fund Support Project of Chinese Medical Association (No. 09010390194) and the Basic and Clinical Research Cooperative project of Capital Medical University (No. 15JL04).

\section{Disclosure}

The authors declare that there are no conflicts of interest.

\section{References}

1. Yang J, Dou G, Tesche C, et al. Progression of coronary atherosclerotic plaque burden and relationship with adverse cardiovascular event in asymptomatic diabetic patients. BMC Cardiovasc Disord. 2019;19 (1):39. doi:10.1186/s12872-019-1016-4

2. Chen Y, Tao Y, Zhang L, Xu W, Zhou X. Diagnostic and prognostic value of biomarkers in acute myocardial infarction. Postgrad Med J. 2019;95(1122):210-216. doi:10.1136/postgradmedj-2019-136409

3. Prastaro M, Pirozzi E, Gaiblazzi N, et al. Expert review on the prognostic role of echocardiography after acute myocardial infarction. $J$ Am Soc Echocardiogr. 2017;30:431-443. doi:10.1016/j. echo.2017.01.020

4. Amsterdam EA, Wenger NK, Brindis RG, et al.; ACC/AHA Task Force Members; Society for Cardiovascular Angiography and Interventions and the Society of Thoracic Surgeons. 2014 AHA/ACC guideline for the management of patients with non-ST-elevation acute coronary syndromes: executive summary: a report of the American College of Cardiology/American Heart Association Task Force on Practice Guidelines. Circulation. 2014;130(25):2354-2394. doi:10.1161/CIR.0000000000000133

5. Prasad SB, Guppy-Coles KB, Holland D, et al. Echocardiographic predictors of all-cause mortality in patients with left ventricular ejection fraction $>35 \%$ : value of guideline based assessment of diastolic dysfunction. Int J Cardiol Heart Vasc. 2019;24:100407. doi:10.1016/j. ijcha.2019.100407

6. Roffi M, Patrono C, Collet JP, et al.; ESC Scientific Document Group. 2015 ESC Guidelines for the management of acute coronary syndromes in patients presenting without persistent ST-segment elevation: task Force for the Management of Acute Coronary Syndromes in Patients Presenting without Persistent ST-Segment Elevation of the European Society of Cardiology (ESC). Eur Heart $J$. 37;2016:267-315. doi:10.1093/eurheartj/ehv320

7. Siddiqui AJ, Holzmann MJ. Association between reduced left ventricular ejection fraction following non-ST-segment elevation myocardial infarction and long-term mortality in patients of advanced age. Int J Cardiol. 2019:pii: S0167-5273(19)32530-6.
8. Alfonso F, Sionis A, Bueno H, et al.; Expert Reviewers for the 2017 ESC Guidelines for the Management of Acute Myocardial Infarction in Patients Presenting with ST-segment Elevation; SEC Guidelines Committee. Comments on the 2017 ESC Guidelines for the Management of Acute Myocardial Infarction in Patients Presenting With ST-segment Elevation. Rev Esp Cardiol (Engl Ed). 70;2017:1039-1045. doi:10.1016/j.recesp.2017.10.049

9. Møller JE, Hillis GS, Oh JK, Reeder GS, Gersh BJ, Pellikka PA. Wall motion score index and ejection fraction for risk stratification after acute myocardial infarction. Am Heart J. 2006;151:419-425. doi:10.1016/j.ahj.2005.03.042

10. Hernesniemi JA, Sipilä K, Tikkakoski A, et al. Cardiorespiratory fitness and heart rate recovery predict sudden cardiac death independent of ejection fraction. Heart. 2019:pii: heartjnl-2019-315198.

11. Schwammenthal E, Adler Y, Amichai K, et al. Prognostic value of global myocardial performance indices in acute myocardial infarction: comparison to measures of systolic and diastolic left ventricular function. Chest. 2003;124(5):1645-1651. doi:10.1378/chest.124.5.1645

12. Baron T, Flachskampf FA, Johansson K, Hedin EM, Christersson C. Usefulness of traditional echocardiographic parameters in assessment of left ventricular function in patients with normal ejection fraction early after acute myocardial infarction: results from a large consecutive cohort. Eur Heart J Cardiovasc Imaging. 2016;17:413-420. doi:10.1093/ehjci/jev160

13. Carluccio E, Tommasi S, Bentivoglio M, Buccolieri M, Prosciutti L, Corea L. Usefulness of the severity and extent of wall motion abnormalities as prognostic markers of an adverse outcome after a first myocardial infarction treated with thrombolytic therapy. $\mathrm{Am}$ J Cardiol. 2000;85:411-415. doi:10.1016/S0002-9149(99)00764-X

14. Trambaiolo P, Bertini P, Borrelli N, et al. Evaluation of ventriculo-arterial coupling in ST elevation myocardial infarction with left ventricular dysfunction treated with levosimendan. Int J Cardiol. 2019;288:1-4. doi:10.1016/j.ijcard.2019.04.052

15. Zhang M, Yang J, Ma C, Liu M. Longitudinal strain measured by two-dimensional speckle tracking echocardiography to evaluate left ventricular function in patients with myocardial bridging of the left anterior descending coronary artery. Echocardiography. 2019;36 (6):1066-1073. doi:10.1111/echo.14357

16. Nagueh SF, Smiseth OA, Appleton CP, et al. Recommendations for the evaluation of left ventricular diastolic function by echocardiography: an update from the American Society of Echocardiography and the European Association of Cardiovascular Imaging. Eur Heart J Cardiovasc Imaging. 2016;17:1321-1360. doi:10.1093/ehjci/jew082

17. Nagueh SF, Smiseth OA, Appleton CP, et al. Recommendations for the evaluation of left ventricular diastolic function by echocardiography: an update from the American Society of Echocardiography and the European Association of Cardiovascular Imaging. $J$ Am Soc Echocardiogr. 2016;29:277-314. doi:10.1016/j.echo.2016.01.011
International Journal of General Medicine

\section{Publish your work in this journal}

The International Journal of General Medicine is an international, peer-reviewed open-access journal that focuses on general and internal medicine, pathogenesis, epidemiology, diagnosis, monitoring and treatment protocols. The journal is characterized by the rapid reporting of reviews, original research and clinical studies
Dovepress

across all disease areas. The manuscript management system is completely online and includes a very quick and fair peer-review system, which is all easy to use. Visit http://www.dovepress.com/ testimonials.php to read real quotes from published authors. 